Chirurg 2020 $91: 778$

https://doi.org/10.1007/s00104-020-01251-2

Online publiziert: 29. Juli 2020

(c) Springer Medizin Verlag GmbH, ein Teil von Springer Nature 2020

\section{Originalpublikation}

Valdimarsson VT, Syk I, Lindell G et al (2020) Outcomes of simultaneous resections and classical strategy for synchronous colorectal liver metastases in sweden: a nationwide study with special reference to major liver resections. World J Surg 2020, https://doi.org/ 10.1007/s00268-020-05475-5

Einleitung. Das kolorektale Karzinom stellt weltweit die dritthäufigste Tumorentität dar. Bereits zum Diagnosezeitpunkt liegen beica. 15-20\% der Patienten Lebermetastasen vor. Die hier vorliegende Arbeit beschäftigte sich mit der Frage, ob es einen Unterschied hinsichtlich des Überlebens zwischen dem Ansatz einer simultanen Resektion gegenüber dem eines sequenziellen, klassischen Vorgehens gibt. Die ausgewerteten Daten entstammen zwei schwedischen Registern. Zum einem dem Swedish Colorectal Cancer Registry und zum anderen dem National Quality Registry for Liver, Bile Duct and Gallbladder Cancer.

Methodik. In dieser prospektiven Registerstudie wurden für den Zeitraum von 2008 bis 2015 insgesamt 537 Patienten herausgefiltert. Von diesen Patienten erhielten 160 eine simultane Resektion des Primus und der Lebermetastasen und 377 wurden einem klassischen Vorgehen unterzogen. Das Überleben sowie patientenspezifische Daten der beiden Gruppen wurden miteinander verglichen. Ausgeschlossen wurden Patienten,

Claudius Gebhardt · Michael Ardelt ${ }^{1}$. Utz Settmacher

${ }^{1}$ Klinik für Allgemein-, Viszeral- und Gefäßchirurgie, Jena, Deutschland

\title{
Ergebnisse nach simultaner Resektion und klassischer Strategie bei synchron hepatisch metastasiertem kolorektalem Karzinom
}

bei denen aufgrund der Tumorerkrankung eine Notfalloperation notwendig war.

Ergebnisse. Bei einem medianen Follow-up von 41 Monaten ergaben die Kaplan-Meier-Kurven eine 5-JahresÜberlebenserwartung nach Diagnosezeitpunkt von $54 \%$ in der Gruppe der Patienten mit klassischem Ansatz und $46 \%$ bei den simultan resezierten Patienten. Hinsichtlich der Major-Leberresektionen zeigte sich kein signifikanter Unterschied das Überleben betreffend in beiden Kohorten bei 155 „klassisch“ und 25 simultan major-resezierten $\mathrm{Pa}$ tienten. Bei simultaner Resektion traten signifikant häufiger Komplikationen auf. Dennoch war der Krankenhausaufenthalt insgesamt sowohl mit als auch ohne Komplikationen kürzer als bei klassisch operierten Patienten. Bei den simultan resezierten Patienten lagen nur in 5\% der Fälle ein Rektumkarzinom als Primum gegenüber $33 \%$ bei den zweizeitig operierten vor.

\section{Kommentar}

Anhand der hier untersuchten Patientendaten konnte kein signifikanter Unterschied bezüglich der 5-Jahres-Überlebenserwartung zwischen den beiden Therapieansätzen festgestellt werden. Da bei den simultan resezierten Patienten in weniger Fällen ein Rektumkarzinom als Primum vorlag und weniger Major-
Leberresektionen durchgeführt wurden, liegt nahe, dass diese Aspekte einflussreiche Selektionskriterien für die patientenindividuelle Therapieplanung darstellen. Somit stellt die simultane Resektion des kolorektalen Primums und der synchronen Lebermetastasen eine hinsichtlich des Überlebens vertretbare Therapieoption im Vergleich zum klassischen Vorgehen unter Inkaufnahme eines erhöhten Komplikationsrisikos dar. Eine Schwäche der hier vorliegenden Arbeit ist sicherlich, dass anhand der verwendeten Register keine Aussage über das rezidivfreie Überleben getroffen werden kann. Ebenso unberücksichtigt bleiben adjuvante Chemotherapien.

\section{Korrespondenzadresse}

PD Dr. med. habil. Dr. med. univ. Michael Ardelt

Klinik für Allgemein-, Viszeral- und Gefäßchirurgie

Am Klinikum 1, 07747 Jena, Deutschland Michael.Ardelt@med.uni-jena.de

Interessenkonflikt. C. Gebhardt, M. Ardelt und U. Settmacher geben an, dass kein Interessenkonflikt besteht. 